\title{
A Micromachined Kelvin Probe With Integrated Actuator for Microfluidic and Solid-State Applications
}

\author{
Larry L. Chu, Kenichi Takahata, Ponnambalam Ravi Selvaganapathy, Yogesh B. Gianchandani, Senior Member, IEEE,
} and J. Leon Shohet, Fellow, IEEE

\begin{abstract}
This paper reports on a micromachined Kelvin probe structure with an integrated scanning tip and an integrated electrothermal actuator that provides axial dithering motion. The device is fabricated from metal foil by a modified microelectrodischarge machining process that allows electrical isolation within the device. In particular, it permits the incorporation of a wide epoxy plug that creates an insulating gap with low parasitic capacitance between the probe and the actuator. The epoxy structures are found to withstand the thermal and mechanical conditions encountered during device operation. The device is used to measure changes in the external surface potential of a parylene microfluidic channel as a function of varying $\mathrm{pH}$ of liquid inside the channel. A contact potential difference of $\approx 6 \mathrm{~V}$ is measured for a change in pH from 4 to 8 within the channel. The device is also used to map embedded charge in a thin $\mathrm{SiO}_{2}$ layer on a $\mathrm{Si}$ substrate, showing it to be suitable for monitoring microelectronics manufacturing processes.

[1072]
\end{abstract}

Index Terms-Contact potential, oxide charge, $\mathrm{pH}$, surface potential.

\section{INTRODUCTION}

$\mathbf{T}$ HE VIBRATING Kelvin probe is an effective and noninvasive tool for the mapping of surface potential of surfaces [1], [22], [26]. Since surface potential includes a component due to work function and another due to trapped charge, this tool can be used to map either quantity on a surface when the other is kept uniform. For example, trapped charge is monitored in semiconductor integrated circuits (IC)-fabrication because it

Manuscript received June 24, 2003; revised December 2, 2004. The work of Y. B. Gianchandani was supported in part by a National Science Foundation Career Award. This work was supported in part by the National Science Foundation by Grant DMR-0084402 and performed in part at the University of Wisconsin Synchrotron Radiation Center. Subject Editor Y.-C. Tai.

L. L. Chu was with the University of Wisconsin, Madison, WI 53706 USA and with the University of Michigan, Ann Arbor, MI 48109 USA. He is now with Colibrys, Inc., Stafford, TX 77477 USA (e-mail: LarryLchu@ gmail.com).

K. Takahata was with the University of Michigan, Ann Arbor, MI 48109 USA. He is now with 3M Corporation, St. Paul, MN 55144 USA.

P. R. Selvaganapathy was with the University of Michigan, Ann Arbor, MI 48109 USA. He is now with the Department of Mechanical Engineering, McMaster University, Hamilton, ON L8S 4L8, Canada (e-mail: selvaga@ mcmaster.ca)

Y. B. Gianchandani is with the Electrical Engineering and Computer Science Department, University of Michigan, Ann Arbor, MI 48109 USA (e-mail: yogesh@umich.edu).

J. L. Shohet is with the Department of Electrical and Computer Engineering, University of Wisconsin, Madison, WI 53706 USA (e-mail: shohet@ece.wisc.edu).

Digital Object Identifier 10.1109/JMEMS.2005.845453 has been correlated to the degradation of the device parameters. The ability to measure local charge distributed across patterns on production wafers can be a critical asset in predicting yield and longevity of devices. This function can be performed by mapping the contact (or surface) potential difference (CPD) between a probe and the sample wafer [14]. Since the Kelvin probe method provides a noncontact and nondestructive diagnostic, it can be used to monitor processes that are known to introduce trapped charges in wafers, such as plasma etch and deposition, ion implantation, and certain cleaning and wafer drying operations. Conventional plasma damage characterization approaches [9]-[11], are based on electrical or surface analytical techniques, which are not suitable for measuring local charge distributions on patterned wafers.

Another potential application for scanning Kelvin probes is the mapping of surface charge of a bio-fluidic tubular sample [4]. Charge distribution on the wall, acquired during manufacturing or in routine operation, can impact the function and behavior of the fluid in the channel. For example, it has been linked to cell adhesion and clotting of red blood cells on artificial surfaces [21]. A Kelvin probe can be used to map the charge embedded in the wall by scanning the outside of the fluidic channel using the electrolyte in the channel as an electrode. A macroscale Kelvin probe has been used to measure the surface potentials of organic overlayers on poorly conducting liquid substrates [23], and a micromachined device based on this principle has been developed for gas sensing [5]. Further, as will be shown in this paper, under certain circumstances a single point measurement can also be used to provide an indication of the local $\mathrm{pH}$ of the fluid.

Relative measurements of surface charge density have been performed using atomic force microscopy (AFM) in an aqueous electrolytic ambient [7], [13], [25]. When the AFM tip is in close proximity to the sample, the electrostatic force produced by their overlapping electrical double layers is detected by the AFM tip and correlated to charge density. This method is effective for measurements of biological samples in an aqueous environment. In nonaqueous environments, noncontact AFM methods have recently been used for the mapping of surface [19] and interface [17] charge on a semiconductor. However, these experiments require ultrahigh vacuum (UHV) and, in some cases, cooling of the system to liquid He temperature, which limit their application. 


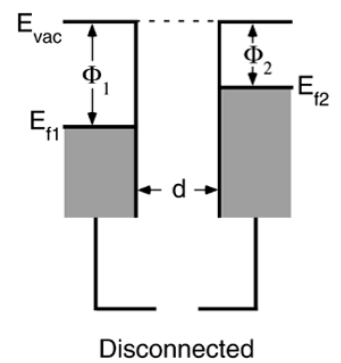

(a)

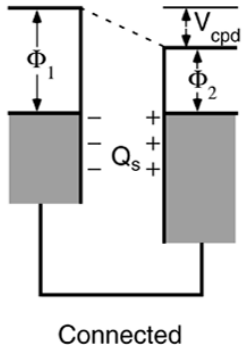

(b)

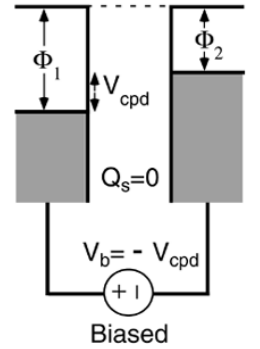

(c)
Fig. 1. Electron energy band diagrams for two different metals separated by an air gap, d. (a-left) before electrical contact (switch is open). (b-middle) after electrical contact (switch is closed). (c-right) after inclusion of the bias potential.

This paper reports on a micromachined Kelvin probe with an integrated scanning tip and actuator. ${ }^{1}$ The actuator provides the axial dither motion, and is electrically isolated from the probe by the wide isolation layer. The device is fabricated from a single sheet of metal foil using a two-step electrodischarge machining process and then bonded to an insulating substrate. This provides a low-cost instrument suitable for measuring microscale samples. Two sets of experiments are used to validate the basic operation of the probe: a semiconductor sample with spatially varying charge in the surface oxide, and a parylene microfluidic channel with varying contents. The device concept and structural design are described in Section II; the microfabrication process is described in Section III, the measurement results are described in Section IV; and Section V provides the conclusions.

\section{DEVICE CONCEPT AND DESIGN}

The Kelvin probe method, which can be applied at room temperature and pressure, uses a vibrating capacitor to measure CPD. The concept is illustrated in Fig. 1. When two disconnected surfaces, such as the tip of a probe and a sample, are brought into proximity, the vacuum levels are aligned [Fig. 1(a)]; and when the two sides are brought into an electrical contact, the Fermi levels are aligned at equilibrium [Fig. 1(b)]. Electron flow is necessary for the equilibrium to be achieved. As a result of this flow, the surface with the larger work function acquires a negative charge, and the other a positive one. An external bias may be used to compensate the electric field resulting from this charge transfer [Fig. 1(c)]. When the system reaches equilibrium, the resulting potential difference is the CPD

$$
V_{\mathrm{CPD}}=\Phi_{12}=\Phi_{1}-\Phi_{2}+\Phi_{D}
$$

where $\Phi_{1}$ and $\Phi_{2}$ are the work functions of the sample and the probe tip, respectively, and $\Phi_{D}$ is the potential due to trapped charge that may exist on their surfaces. As the probe, which is biased at $-V_{b}$ with respect to the sample, is dithered in close proximity to it, an ac current is induced by the modulation of the capacitance $\left(C_{K}\right)$ between them

$$
i=\left(V_{\mathrm{CPD}}+V_{b}\right) \cdot\left(\frac{d C_{K}}{d t}\right) .
$$

${ }^{1}$ Portions of this manuscript have appeared in conference abstract form in [8].
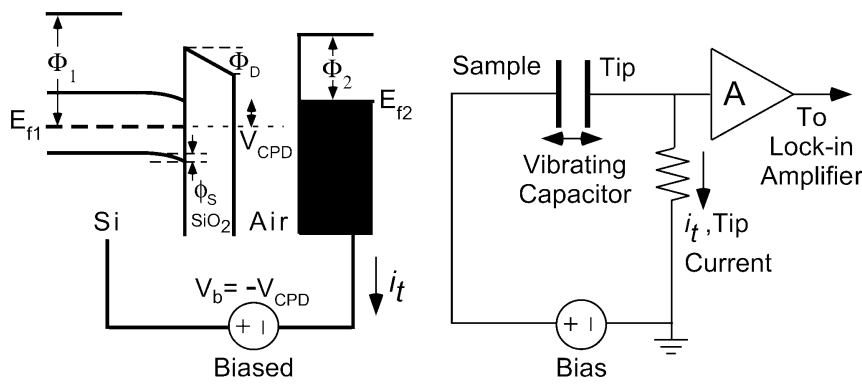

Fig. 2. The energy band diagram for a charged oxide on Si system with applied bias that results in zero electric field in the air gap.

Theoretically, $V_{b}$ is varied until the current goes to zero to determine $V_{\mathrm{CPD}}$. In practice, the magnitude of the current does not go to zero and is affected not only by noise, but by the finite input impedance and the leakage currents of the test instruments as well as parasitic pathways in the device and setup. Thus, in practice, the rms current is monitored, and the bias that minimizes it is $-V_{\mathrm{CPD}}$.

The energy band diagram for the CPD measurement of a semiconductor sample with a metal probe is shown in Fig. 2. In this situation there is an additional potential drop of $\phi_{S}$ in the semiconductor, which must be added to the right-hand side (RHS) of (1). However, the magnitude of $\phi_{S}$ is relatively small in most situations. The CPD is also affected by any charges that might be trapped in the system, e.g., within the oxide or at the oxide-semiconductor interface. A simple analogy is to the flat-band voltage $\left(V_{F B}\right)$ of an MOS transistor (for which $\phi_{S}$ is zero) [20], [27]

$$
V_{F B}=\Phi_{12}-\frac{Q_{s s}}{C_{\mathrm{ox}}}-\frac{1}{C_{\mathrm{ox}}} \int_{0}^{x_{\mathrm{ox}}} \frac{x}{x_{\mathrm{ox}}} p(x) d x
$$

where $C_{\mathrm{ox}}$ is the capacitance per unit area due to the oxide layer on semiconductor, $x_{\mathrm{ox}}$ is the thickness of the oxide layer, $p(x)$ is the local charge density distribution through the thickness of the oxide layer, and $Q_{s s}$ is the total charge density per unit are at the oxide-semiconductor interface. It is noteworthy that the interface charge density is due to traps that are distributed in energy across the bandgap in the semiconductor, so the magnitude of this term varies with the Fermi level. This means that it can vary with the doping concentration in the semiconductor, the temperature, and $\phi_{S}$. While it also varies with applied bias voltage, this dependence does not affect (2a), which is specifically for the flat-band condition. However, the charging and discharging of interface traps can certainly distort the relationship between the overall capacitance and applied bias.

The work function for a semiconductor is given by

$$
\Phi_{\text {semic }}=\chi+\frac{k T}{q} \ln \left(\frac{N_{C}}{N_{d}}\right)
$$

where $\chi$ is the electron affinity of the semiconductor, $T$ is the absolute temperature, $k$ is Boltzmann's constant, $q$ is magnitude of charge on an electron, $N_{C}$ is the effective density of states at the conduction band edge, and $N_{d}$ is the dopant concentration, assuming that the material is not degenerately doped. The dopant concentration affects the Fermi level, and through that, the work function. 
The situation for a liquid under a dielectric is quite similar to that of the semiconductor under oxide. The work function for the liquid is given by [6]

$$
\Phi_{\text {liq }}=\chi A z_{i}+\left(\frac{A k T \ln \left(f_{i} c_{i}\right)+\mu_{i}^{0}}{q}\right)
$$

where $A$ is Avogadro's number; $z_{i}$ is valence on the ion species of interest in the liquid; $\mu_{i}^{0}$ is the standard chemical potential for unity activity, $a_{i}$, which is the product of the activity coefficient, $f_{i}$, and concentration, $c_{i}$, of the ion species. (In dilute solutions, $f_{i} \approx 1$.) The variation in ionic concentration provides a nominal deviation of $59 \mathrm{mV} / \mathrm{dec}$ that is quite similar to the variation in semiconductor work function due to doping level, as expressed in (2b). As for semiconductors, a number of complicating factors must be considered, although the nature of the complexities and their relative contributions can differ. For example, interfering ions, which tend to be a greater challenge for liquid samples than for solid-state samples, cause a deviation from the value of the work function predicted by (2c). The CPD measurement for a liquid is further affected by variations in the other terms in (2c) that control the work function. In addition, variations in concentration can affect the CPD indirectly, in a manner analogous to the interface traps at the semiconductor-oxide interface. For example, zeta potential, which is to some extent analogous to $\phi_{S}$ in that it represents the potential drop from the proximity of the wall into the depth of the sample, is affected by both $\mathrm{pH}$ and concentration [16]. In addition, the surface charge that the polymer wall acquires due to its interaction with the electrolyte in the microchannel is dependent on $\mathrm{pH}$ among other factors. In general, protonation, deprotonation, adsorption and other reaction equilibria define a net charge density on the surface. The mechanism for the establishment of surface charge is an area of active investigation [16]. Typically, the surface charge density is calculated using the following relation for zeta potential data, which is obtained in turn from electroosmotic and streaming potential measurements [12]

$$
\sigma_{C}=\sqrt{8 k T \varepsilon_{a q} n^{0}} \sinh \left(\frac{z q \phi_{0}^{C}}{2 k T}\right)
$$

and $\phi_{0}^{C}=(\zeta / \exp (-\kappa \gamma)) ; \kappa=\sqrt{\left(2 n^{0} z^{2} q^{2} / \varepsilon_{a q} k T\right)}$ where $\sigma_{c}$ is the surface charge density; $\varepsilon_{a q}$ is the permittivity of the buffer; $\phi_{0}^{C}$ is the potential drop across the double layer; $n^{0}$ is the number concentration of the electrolyte; $\zeta$ is the zeta potential; $\gamma$ is the thickness of the inner Helmholtz layer that determines the zeta potential; and $\kappa$ is the inverse of the diffuse layer thickness. A number of researchers have reported that the zeta potential changes linearly with $\mathrm{pH}$ over the range of $2-8 \mathrm{pH}$ [16]. Further, zeta potential is proportional to the surface charge density as $\sinh (x)$ in (2d) can be approximated by $x$ for small values of $x$. As will be shown in Section IV, this is consistent with the measured results obtained in this effort.

From this discussion, it can be seen that in most practical situations, theoretical predictions of CPD from first principles can be a challenge because the values of all the variables are not available. Despite this, CPD measurements can provide significant value in many contexts, particularly as empirical standards are established and used in monitoring processes that have narrow tolerances.

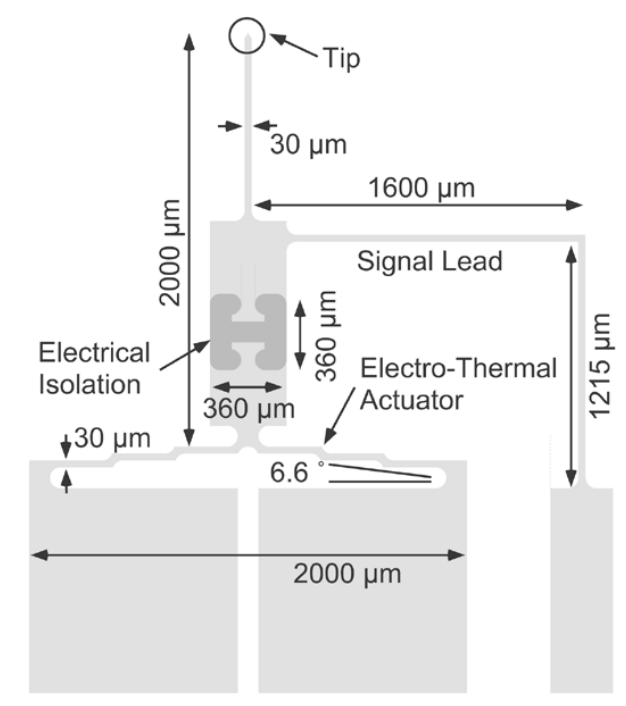

Fig. 3. Schematic of the $\mu$ EDM Kelvin probe device showing the dimensions of the structure.

\section{A. Device Design}

The reported device includes an actuator that provides the axial dither motion, the sense probe, which is electrically isolated from the actuator, and a lead transfer beam for the probe (Fig. 3). A wide isolation region is essential to minimize capacitive coupling between the drive signal of the actuator and probe. An insulating glass substrate also helps in this regard, as does the choice of actuator. The bent-beam actuator is electrothermally driven by passing current through the bent beam, which amplifies the resulting deformation into an outward motion of the tip [24]. It is selected because it offers nonresonant dither motion with amplitude in the $10-\mu \mathrm{m}$ range with drive voltages of a few volts. The low voltage minimizes the coupling of the drive signal to the sense probe, while the large amplitude nonresonant displacement permits the dithering frequency and amplitude to be varied to suit the needs of the measurement. For measurements made along micromachined capillary channels, the electrical ground is provided by the conductive fluid present in the channel. However, the low conductivity of the liquid, and the long and narrow shape of the channel, can make the access resistance to the point of measurement large, and create a slow $R C$ time constant. To accommodate this, the dither frequency of the Kelvin probe is kept low, sacrificing sense current amplitude.

In some situations it might be appropriate to have a laterally symmetrical layout for the lead transfer from the probe (Fig. 3), so that there is no net lateral force on the probe as it is pushed forward by the actuator. One possibility is to replicate the attachment that is presently used so that there lateral symmetry on the two sides of the shank. This would, however, increase the loading force on the actuator and create the need for a stronger drive signal. In addition, if traditional electrodischarge machining is used (as will be discussed in Section III), the presence of the additional beam can increase the machining time. In this effort, only the asymmetrical layout has been fabricated and tested.

Miniaturization of a Kelvin probe structure can potentially increase $\mathrm{kT} / \mathrm{C}$ noise from the reduction in sense capacitance. When the signal is weak, the noise from the sense electronics 
(A)

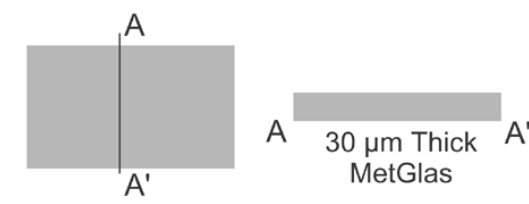

(B)

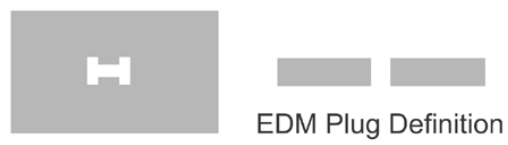

(C)
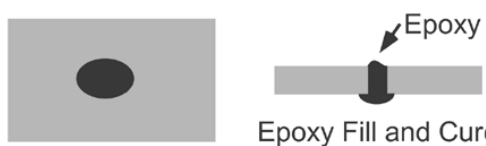

Epoxy Fill and Cure

(D)
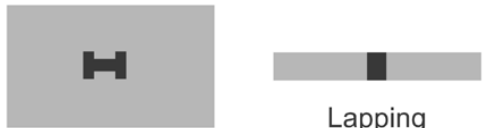

Lapping

$(\mathrm{E})$

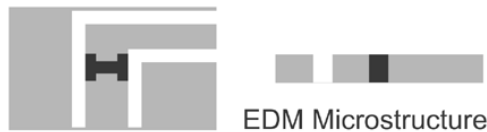

(F)

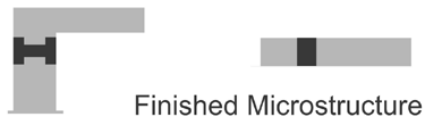

Fig. 4. The micro EDM fabrication sequence to allow dielectric plugs and electrical isolation.

can also be relatively significant. Another potential source of noise is the thermomechanical vibration of the probe (although this will be relatively small in most cases). To some extent the increase in noise is an essential compromise for spatial resolution and the overall size advantage.

\section{FABRICATION}

The micromachined Kelvin probe is fabricated by a modified microelectrodischarge machining ( $\mu \mathrm{EDM})$ process. This technique is attractive because it can be used to fabricate parts from any electrically conductive material. Batch mode $\mu \mathrm{EDM}$, performed with electroplated arrays of electrodes, offers very high throughput [28]. However, normal $\mu \mathrm{EDM}$ by itself does not allow electrical isolation since all the mechanically connected features are all electrically connected. In the case of the micromachined Kelvin probe, the probe tip must be isolated from the dithering actuator, preferably by a large width of isolation to minimize the capacitive feedthrough of the drive signal [2].

The starting material is a commercially available $30 \mu \mathrm{m}$ thick stock metal sheet of MetGlas 2826MB; it is an alloy of primarily $\mathrm{Ni}$ and Fe [Fig. 4(a)]. First, a traditional $\mu$ EDM step is performed to define the shape of the epoxy plug in the work piece [Fig. 4(b)]. The piece is removed from the $\mu \mathrm{EDM}$ oil bath and cleaned in an ultrasonic bath with detergent. A quick-hardening two-part epoxy is properly mixed and applied onto the machined work piece to fill the plug [Fig. 4(c)]. The epoxy is allowed to

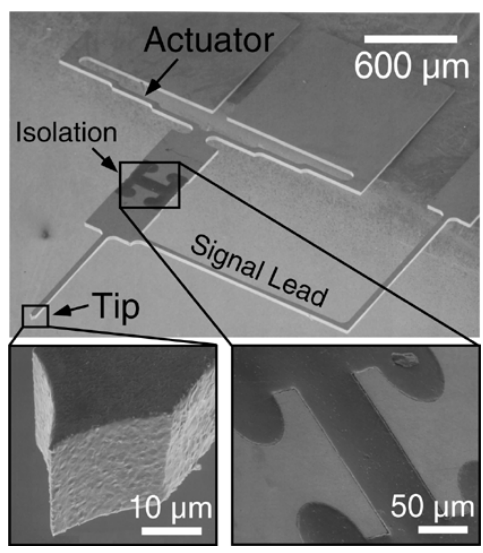

Fig. 5. SEM picture of the device and the close-up pictures of the tip area and the isolation plug.

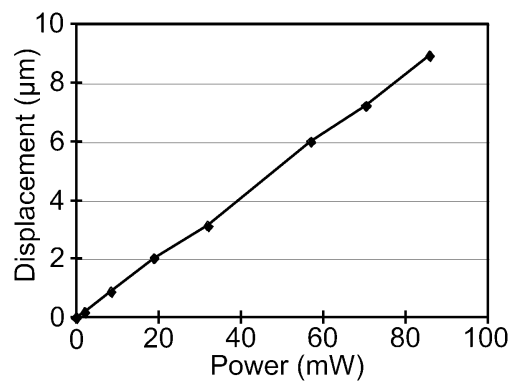

Fig. 6. The response of the electrothermal actuator.

cure for over $12 \mathrm{~h}$ to achieve proper mechanical stiffness and adhesion. A lapping step is performed for both sides of the work piece to remove the excess cured epoxy [Fig. 4(d)]. The work piece is cleaned before returning to the $\mu \mathrm{EDM}$ machine for the definition of the rest of the microstructure with the rest of the features aligned to the epoxy plug, which is released by cutting along its edges [Fig. 4(e)]. Finally, the finished part is attached to a substrate for testing [Fig. 4(f)]. A glass substrate is used to minimize parasitic capacitance that might cause the sensed signal to leak away. The finished structure is shown in Fig. 5. In a variant of the device, a larger Kelvin probe tip is attached onto the micromachined device for sample which has coarse spatial variation and weak Kelvin probe signals. The larger tip has an area of $0.01 \mathrm{~mm}^{2}$ and is made of tungsten. Also shown in Fig. 5 in the inserts is a close up of the tip area and the epoxy-filled isolation.

\section{EXPERIMENTAL RESULTS}

The operation of the integrated electrothermal actuator was verified by driving it with a supplied current while monitoring the displacement of the tip by a calibrated optical method. The actuator generated a maximum displacement of $9 \mu \mathrm{m}$ when actuated at $85 \mathrm{~mW}$ with a resistance of $4.2 \Omega$ (Fig. 6), satisfying the displacement requirements. (For this device, $<5 \mu \mathrm{m}$ of displacement was needed to generate a Kelvin probe signal.)

The Kelvin probe provides a very small current signal, which can be a challenge to measure. If a parallel plate approximation 


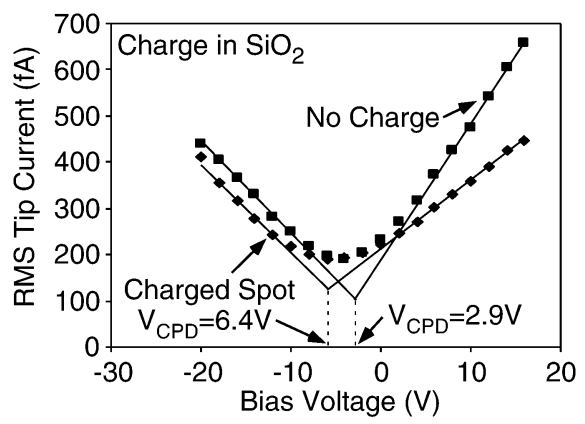

Fig. 7. Two Kelvin probe traces that demonstrates how the rms tip current is interpreted to obtain the CPD.

is used for the capacitance between the probe and sample, the signal amplitude is

$$
\begin{aligned}
i & =\left(V_{\mathrm{CPD}}+V_{b}\right) \cdot \frac{d C_{K}}{d t} \\
& =2 \cdot f \cdot\left(V_{\mathrm{CPD}}+V_{b}\right) \cdot \varepsilon \cdot A \cdot\left(\frac{1}{g_{0}-g_{a}}-\frac{1}{g_{0}}\right)
\end{aligned}
$$

where $f$ is the actuation frequency, $\varepsilon$ is the permittivity of the medium (air), $A$ is the tip capacitor area, $g_{0}$ is the initial capacitor gap, and $g_{a}$ is the actuation displacement. Assuming a tip area of $0.01 \mathrm{~mm}^{2}, V_{\mathrm{CPD}}=1 \mathrm{~V}$, an actuation frequency of $13 \mathrm{~Hz}$, and a capacitive gap of $5 \mu \mathrm{m}$ (with $50 \%$ gap modulation due to actuation), the tip current is in the order of $500 \mathrm{fA}$. Hence, it is important to minimize noise from the measurement setup and electronics. The current signal was fed through a transconductance amplifier (SR570) set at $1 \mathrm{~V} / \mathrm{pA}$ and then detected with a lock-in amplifier (SR830 DSP) that was frequency locked to the actuation frequency. The current sensing resistance shown in Fig. 2 is inside the instrument, which has a virtual ground at its input and an equivalent input impedance of $1 \mathrm{M} \Omega$; using an actual resistor for current sensing would have produced a potentially unacceptable level of Johnson noise due to the large resistance necessary to sense such small currents. The actuation frequency was selected to avoid harmonics and subharmonics of common noises such as $60-\mathrm{Hz}$ power line interference. The Kelvin probe signal is maximized when the tip-to-sample average gap is minimized and when the dithering amplitude is maximized. Given the displacement characteristics of the integrated actuator, an average gap of $\sim 5 \mu \mathrm{m}$ with a dithering amplitude of 2-4 $\mu \mathrm{m}$ was selected. Potential harmonic distortion of the output signal is mitigated by the use of a lock-in amplifier. The lock-in amplifier essentially provides the rms probe current [3]. The measured rms probe current as a function of $V_{b}$ is compared for two locations on a charged sample in Fig. 7. These results were obtained with a tungsten tip with a sense area of $0.01 \mathrm{~mm}^{2}$ and a $13-\mathrm{Hz}$ dither. As shown, the $V_{\mathrm{CPD}}$ must be extrapolated from tangents drawn to the response curve at high bias values, because at low currents the amplifier noise and parasitic feed through from the drive signal can be significant. Essentially, the expected triangular-shaped response of rms current magnitude is superimposed on a noise floor of approximately 150-200 fA. When the tip-to-sample separation is changed, the slope of the current-to-bias response curve changes (Fig. 8). Measurement artifacts from this are eliminated by keeping the tip-sample separation constant during a scan. Generally, this

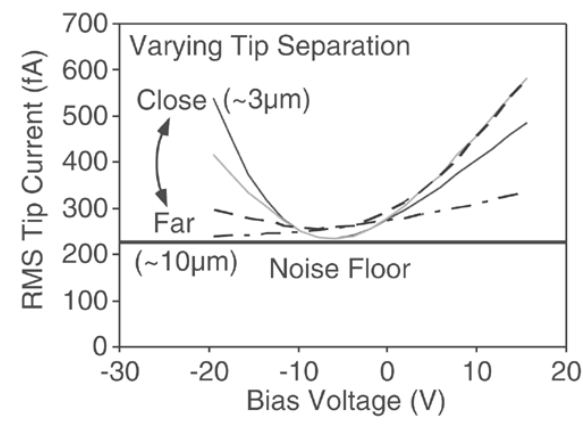

Fig. 8. The Kelvin probe signal obtained at different gap separation; this result indicates that when the tip is too far, the signal is not reliable.

means that the slope of the rms tip current to $V_{b}$ should be similar for all readings.

Two sets of experiments were used to validate the basic operation of the probe: a semiconductor sample with spatially varying charge in the surface oxide, and a parylene microfluidic channel with varying contents. In one, the probe was used to provide a spatial map of charge distribution of a sample that was otherwise uniform and invariant over the period of observation; in the other, the probe was held at a fixed location over a sample, and a single point measurement was taken for differing channel contents.

The use of the Kelvin probe to map charge distribution over a sample surface area can be useful in a number of contexts, particularly in assessing plasma implantation and vacuum ultraviolet (VUV) photoemission. In order to evaluate the capability of the fabricated Kelvin probes in this regard, a test wafer was exposed to VUV radiation from a synchrotron to mimic charging due to photoemission during IC fabrication, as shown in Fig. 9(a). Fig. 9(b) shows the areal distribution of $V_{\mathrm{CPD}}$ on an Si sample with 359-nm-thick oxide to VUV radiation. Spatial variation of $V_{\mathrm{CPD}}$ from 1 to $7 \mathrm{~V}$ over a few hundred microns is evident. The position of the peak corresponds to the center of the VUV exposure.

The measurement setup for the microfluidic tests is shown in Fig. 10. Electrical access to the interior of the channel was provided through an Au electrode that was in contact with the fluid inside the channel, but did not extend along its full length. The electrical equivalent circuit is shown in Fig. 11: the capillary wall capacitance is $C_{w}$, the Kelvin probe sense capacitance is $C_{K}$, while $R_{C}$ and $C_{C}$ are due to the coaxial cable from the probe tip to the amplifier, which has an input impedance of $R_{A}$ and $C_{A}$. Also, $R_{B}$ is the internal resistance of the biasing dc source together with the cable resistance from the biasing source to the conductor inside the channel. These components are not affected when the $\mathrm{pH}$ of the liquid in the channel is changed. On the other hand, several components are potentially affected by change in $\mathrm{pH}$. These include $Z_{D 1}$ (composed of $R_{D 1}, C_{D 1}$ ) and $Z_{D 2}$ (composed of $R_{D 2}, C_{D 2}$ ), the impedances of the double layers at the electrolyte-metal and electrolyte-polymer interfaces [15], respectively. Also affected is the resistance of the fluid between the two double layers, $R_{F}$, although the impact of this is diminished in comparison to the much larger impedance due to the Kelvin probe capacitance, $C_{K}$. Due to the large input impedance of the amplifier, the cable 


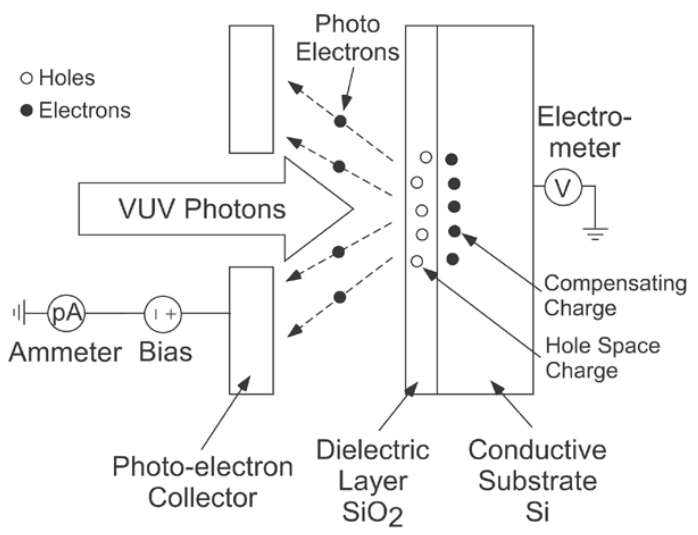

(a)

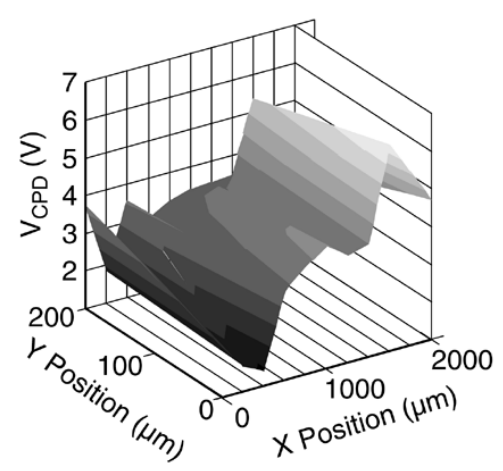

(b)

Fig. 9. (a) Charging during IC fabrication emulated by exposing a Si wafer with 359-nm-thick oxide to vacuum UV synchrotron radiation. (b) Areal map of the resulting $V_{\mathrm{CPD}}$ showing a portion of the exposed region.

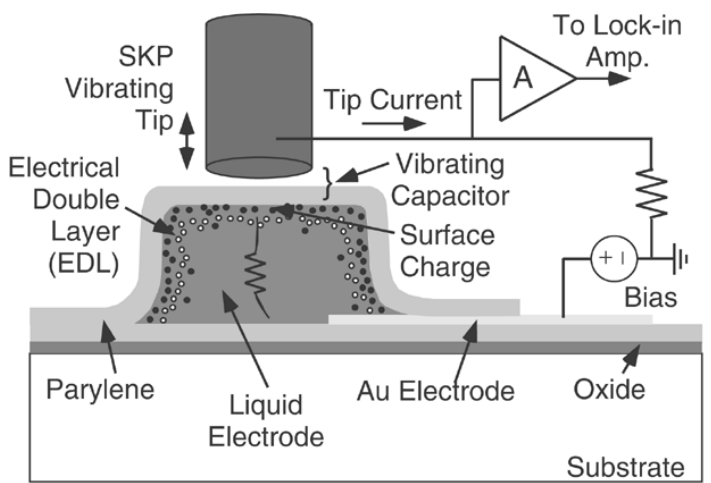

(a)

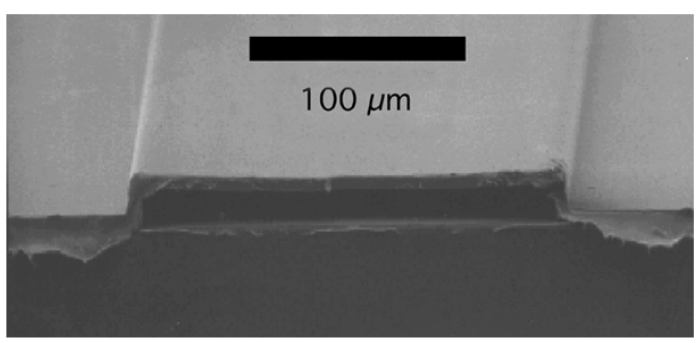

(b)

Fig. 10. (a) Measurement setup for microfluidic surface potential using Kelvin probe. (b) The microfluidic channel used in the measurements had $3-5-\mu \mathrm{m}$-thick parylene walls and was $20 \mu \mathrm{m}$ high [18].

resistances, $R_{B}, R_{C}$ are relatively small. Of course, all these potential changes that can take place because of the change in $\mathrm{pH}$ are in addition to those discussed in Section II.

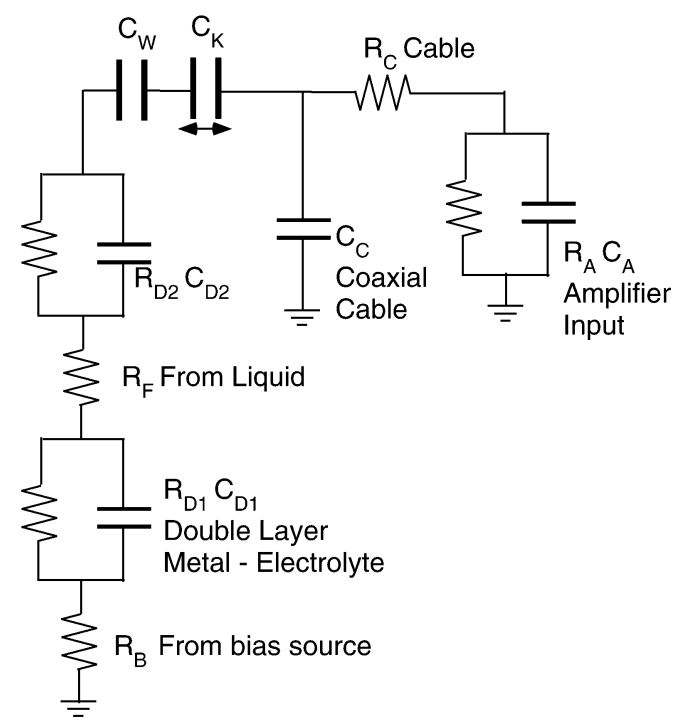

Fig. 11. The equivalent circuit for the microfluidic channel measurement using the micromachined Kelvin probe.

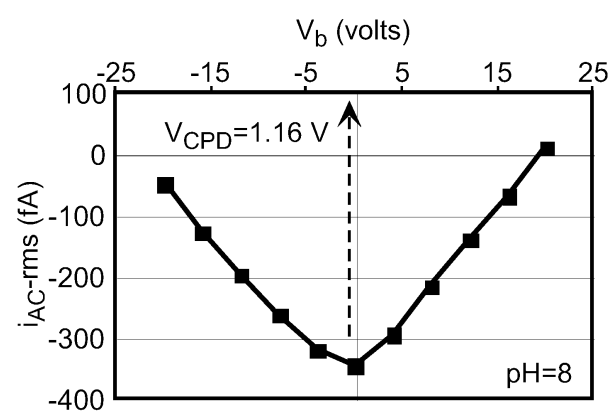

(a)

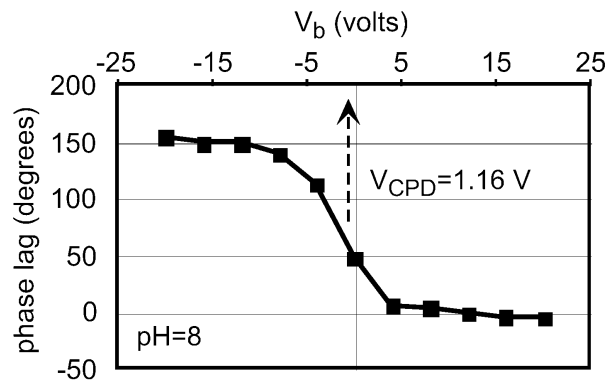

(b)

Fig. 12. (a) Measured sense current amplitude. (b) Phase at a single spot above the microchannel with a tip-sample spacing of $\approx 5 \mu \mathrm{m}$ and dither amplitude of $\approx 2 \mu \mathrm{m}$. Both plots contain offset values which are inconsequential.

Experiments were performed to determine relationship between the $V_{\mathrm{CPD}}$ above a single point of the microchannel and the $\mathrm{pH}$ of the solution within it. The measured sense current for a pH 8 buffer is shown in Fig. 12. Despite the offset caused by leakage currents and other nonidealities (as discussed in Section II), the minimum of the curve that corresponds to $-V_{\mathrm{CPD}}$ is evident in the current magnitude plot of Fig. 12(a). It is notable that the phase response in Fig. 12(b) also shows a sharp transition near this value. As with current magnitude, the phase depends on a variety of factors, including the measurement setup and instrumentation. 


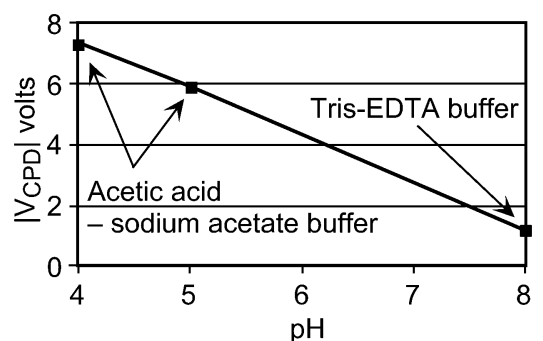

Fig. 13. Measured $V_{\mathrm{CPD}}$ showing linear dependence on $\mathrm{pH}$, which was calibrated using a pH meter (Piccolo, Hanna Instruments) of $0.01 \mathrm{pH}$ resolution and accuracy.

The contact potentials for three different cases are plotted in Fig. 13, showing that the relationship is essentially linear. Acetic acid-sodium acetate and Tris-EDTA buffer systems were used to ensure that the $\mathrm{pH}$ in the channel was uniform and not affected by local residues when the system was flushed. The total concentrations of the solutions for all three $\mathrm{pH}$ measurements were kept constant at $100 \mathrm{mM}$. A contact potential difference of $\approx 6 \mathrm{~V}$ was measured for a change in $\mathrm{pH}$ from 4 to 8 within the channel. This correlates well with observation in Section II that surface charge on the polymeric wall of the channel would have linear dependence on the $\mathrm{pH}$ of solution in the channel. Overall, these measurements suggest that despite the complexity of theoretical predictions and the many unknown variables, under controlled circumstances it may be possible to use CPD measurements for noncontact evaluation of liquids in microchannels.

\section{CONCLUSION}

This paper has demonstrated how a relatively simple design and fabrication process can be used to form a micromachined Kelvin probe with an integrated dither actuator, and further, how the device can be used to provide surface potential measurements for both semiconductor and microfluidic diagnostics. The electrothermal actuator achieves a maximum of $9 \mu \mathrm{m}$ displacement when actuated at $85 \mathrm{~mW}$. The Kelvin probe tip is electrically isolated from the actuator using a process modification that permits wide expanses of dielectric materials to be embedded in the structure. The device was successfully used to measure the two-dimensional charge variation in $\mathrm{SiO}_{2}$ on a $\mathrm{Si}$ substrate and solution variations of $\mathrm{pH}$ in a microfluidic channel. In the latter context, it is possible to envision a microfluidic system with a Kelvin probe integrated above critical positions in the channel, permitting integrated in-line measurements in real time to control chemical reactions.

\section{ACKNOWLEDGMENT}

The authors are grateful to Prof. F. Terry of the University of Michigan and J. Lauer of the University of Wisconsin for helpful discussions. J. Lauer also performed the synchrotron exposures.

\section{REFERENCES}

[1] J. R. Anderson and A. E. Alexander, "Theory of the vibrating condenser converter and application to contact potential measurements," Australian J. Appl. Sci., vol. 3, pp. 201-201, 1952.

[2] I. D. Baikie, E. Venderbosch, J. A. Meyer, and P. J. Estrup, "Analysis of stray capacitance in the Kelvin method," Rev. Sci. Instr., vol. 62, pp. 725-725, 1991.
[3] I. D. Baikie, S. Mackenzie, and P. J. Z. Estrup, "Noise and the Kelvin method," Rev. Sci. Instr., vol. 62, pp. 1326-1332, 1991.

[4] I. D. Baikie, P. J. S. Smith, D. M. Porterfield, and P. J. Estrup, "Multi-tip scanning bio-Kelvin probe," Rev. Sci. Instr., vol. 70, no. 3, pp. 1842-1842, 1999.

[5] P. L. Bergstrom, R. Merchant, K. D. Wise, and J. W. Schwank, "Dielectric membrane technology for conductivity and work-function gas sensors," in IEEE Int. Conf. Sens. Actuators (Transducers 1995), Stockholm, Sweden, 1995, pp. 993-996.

[6] P. Bergveld, J. Hendrikse, and W. Olthuis, "Theory and application of the material work function for chemical sensors based on the field effect principle," Meas. Sci. Technol., pp. 1801-1808, 1998.

[7] H.-J. Butt, "Measuring local surface charge densities in electrolyte solutions with a scanning force microscope," Biophys. J., vol. 63, no. 2, pp. 578-582, 1992.

[8] L. L. Chu, K. Takahata, P. Selvaganapathy, J. L. Shohet, and Y. B. Gianchandani, "A micromachined Kelvin probe for surface potential measurements in microfluidic channels and solid-state applications," in IEEE Int. Conf. Sens. Actuators (Transducers 2003), Boston, MA, 2003, pp. 384-387.

[9] C. Cismaru, J. L. Shohet, K. Nauka, and J. B. Friedmann, "Relationship between the charging damage of test structures and the deposited charge on unpatterned wafers exposed to an electron cyclotron resonance plasma," Appl. Phys. Lett., vol. 72, no. 10, pp. 1143-5, 1998.

[10] S. Fang and J. P. McVittie, "Thin-oxide damage from gate charging during plasma processing," IEEE Electron Device Lett., vol. 13, pp. 288-288, 1992.

[11] J. B. Friedmann, J. L. Shohet, R. Mau, N. Hershkowitz, S. Bisgaard, S. Ma, and J. P. McVittie, "Plasma-parameter dependency of thin-oxide damage from wafer charging during electron-cyclotron-resonance plasma processing," IEEE Trans. Semicond. Manuf., vol. 10, no. 1, pp. 154-154, 1997.

[12] N. K. Hartley and M. A. Hayes, "Examination of theoretical models in external voltage control of capillary electrophoresis," Anal. Chem., vol. 74, pp. 1249-1255, 2002.

[13] W. F. Heinz and J. H. Hoh, "Relative surface charge density mapping with the atomic force microscope," Biophys. J., vol. 76, pp. 528-38, 1999.

[14] A. Hoff, K. Nauka, T. Persson, J. Lagowski, L. Jastrzebski, and P. Edelman, "A novel approach to monitoring of plasma processing equipment \& plasma damage without test structures," in IEEE/SEMI Adv. Semicond. Manuf. Conf., 1997, pp. 185-185.

[15] R. J. Hunter, Introduction to Modern Colloid Science. Oxford, U.K.: Oxford Science, 1993.

[16] B. J. Kirby and E. J. Hasselbrink, "Zeta potential of microfluidic substrates: 1 . Theory, experimental techniques, and effects on separations," Electrophoresis, vol. 25, pp. 187-202, 2004.

[17] R. Ludeke and E. Cartier, "Imaging of oxide and interface charges in $\mathrm{SiO}_{2}$-Si," Microelectron. Eng., vol. 59, pp. 259-263, 2001

[18] F. Man, D. K. Jones, and C. H. Mastrangelo, "Microfluidic plastic capillaries on silicon substrates: A new inexpensive technology for bioanalysis chips," in IEEE Int. Conf. Micro Electro Mechan. Syst., Nagoya, Japan, 1997, pp. 311-6.

[19] S. Morita, M. Abe, K. Yokoyama, and Y. Sugawara, "Defects and their charge imaging on semiconductor surfaces by noncontact atomic force microscopy and spectroscopy," J. Crystal Growth, vol. 210, pp. 408-15, 2000.

[20] R. S. Muller and T. I. Kamins, Device Electronics for Integrated Circuits. New York: Wiley, 1977.

[21] P. V. Murphy and S. Merchant, "Blood compatibility of polymer electrets," in Proc. Int. Conf. on Electrets, Charge Storage, and Transport in Dielectrics, Miami Beach, FL, 1973, pp. 627-649.

[22] W. Nabhan and B. Equer, "A high-resolution scanning Kelvin probe microscope for contact potential measurements on the $100 \mathrm{~nm}$ scale," Rev. Sci. Instr., vol. 68, no. 8, pp. 3108-3108, 1997.

[23] I. R. Peterson, "Kelvin probe liquid-surface potential sensor," Rev. Sci. Instr., vol. 70, no. 8, pp. 3418-3424, Aug. 1999.

[24] L. Que, J. S. Park, and Y. B. Gianchandani, "Bent-beam electro-thermal actuators-I: Single beam and cascaded devices," J. Microelectromech. Syst., vol. 10, no. 2, pp. 247-254, 2001.

[25] R. Raiteri, M. Grattarola, and H.-J. Butt, "Measuring electrostatic double-layer forces at high surface potentials with the atomic force microscope," J. Phys. Chem., vol. 100, pp. 16700-5, 1996.

[26] N. A. Surplice and R. J. D' Arcy, "A critique of the Kelvin method of measuring work functions," J. Phys. E: Scientif. Instrum., vol. 3, pp. 477-477, 1970.

[27] S. M. Sze, Physics of Semiconductor Devices. New York: Wiley, 1981.

[28] K. Takahata and Y. B. Gianchandani, "Batch mode micro-electro-discharge machining," J. Microelectromech. Syst., vol. 11, no. 2, pp. 102-10, 2002. 


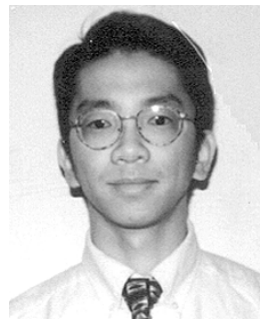

Larry L. Chu received the B.S., M.S., and Ph.D. degrees in electrical engineering from the University of Wisconsin, Madison, in 1998, 2001, and 2003 respectively. His research focused on applications of microactuators, with an emphasis on electrothermal devices. Some of the electrothermally driven devices that he worked on include: rotary and linear geared mechanisms, compliant motion amplification devices, and close-loop precision translation stages. Another aspect of his work involves characterization of materials commonly used in micromachining.

In 2003, he joined the Center for Nanotechnology (CNTech), WI, as a Postdoctoral researcher and concentrated on applying microfluidics to synthesize DNA and genes using a maskless microarray technique. Until 2005, he was with Genetic Assemblies, Inc., a biotechnology start-up that is commercializing the gene synthesis technology, as a Research Scientist. He is currently with Colibrys, Inc., Stafford, TX. His current research interests include micromachined electrothermal actuation applications, microfluidic fabrication, and bio-instrumentation.

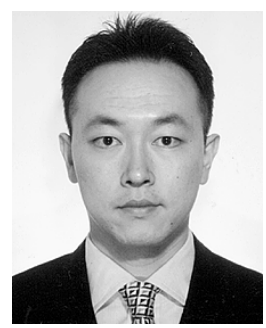

Kenichi Takahata received the B.S. degree in physics from Sophia University, Tokyo, Japan, in 1990 and the M.S. degree in electrical engineering from the University of Michigan, Ann Arbor, in 2004. He received the Ph.D. degree in electrical engineering from the University of Michigan.

In 1990, he joined Matsushita Research Institute Tokyo, Inc. (Panasonic) and was with Matsushita Electric Industrial Co., Japan, until 2001. At Matsushita, he was engaged in R\&D of micromechanics and microfabrication technologies including microelectrodischarge machining ( $\mu$ EDM) partially for the Japanese National Project "Micromachine Technology." In 1997, he was appointed Researcher in the International Joint Research Program supported by New Energy and Industrial Technology Development Organization (NEDO) of Japan, which explored the compatibility between $\mu \mathrm{EDM}$ and deep X-ray lithography (LIGA) processes at the University of Wisconsin-Madison. From 1999 to 2001, he was a Visiting Scientist with the University of Wisconsin-Madison for collaboration on batch mode $\mu \mathrm{EDM}$ technology that utilized lithographically fabricated electrodes and its application to MEMS. Presently, he is with 3M Corporation, St. Paul, MN. He currently has 24 publications, five issued patents, and 23 pending patents in the United States and Japan. His research interests are in MEMS realized by a combination of silicon and nonsilicon based manufacturing technologies.

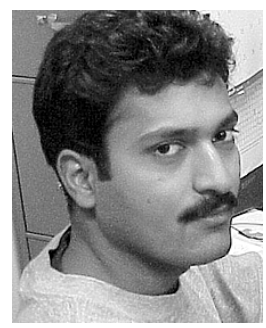

Ponnambalam Ravi Selvaganapathy was born in Madras, India, in 1977. He received the B.S. degree in chemical and electrochemical engineering from Central ElectroChemical Research Institute (CECRI), Karaikudi, India, in 1998. During his research at CECRI, he worked on cathodic disbondment of polymer coatings due to corrosion and on development finite element software for corrosion monitoring and detection. He received the M.S. and $\mathrm{Ph} . \mathrm{D}$. degrees in electrical engineering and computer science from the University of Michigan, Ann Arbor, in 2001 and 2002, respectively. He also received the M.S. degree in nuclear engineering (materials) from the University of Michigan in 2002.

From 1998 through 2002, he was a Research Assistant with the Center for Integrated MicroSystems, University of Michigan, working on a variety of projects related to MEMS and BioMEMS processes and device development including electrochemical detection in capillary electrophoresis systems, electroosmotic pumping systems, prevention of bubble generation in electrically active microfluidic systems, thermal phase change polymers, development of microfabrication techniques for porous polymers in microsystems, development of a contact-free technique for $\mathrm{pH}$ measurement using the Kelvin probe, and investigation of microplasmas as a fluorescence excitation source for DNA. He then worked as a Postdoctoral Research Scientist with Sandia National Laboratories, Livermore, until June 2004, in the area of integrated microfluidic systems. He is presently an Assistant Professor of Mechanical Engineering at McMaster University, Canada.

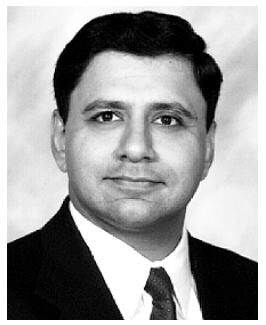

Yogesh B. Gianchandani (S'83-M'85-SM'04) received the B.S., M.S., and after some time in industry, the Ph.D. degrees in electrical engineering, with a focus on microelectronics and MEMS.

$\mathrm{He}$ is presently an Associate Professor with the Electrical Engineering and Computer Science Department, University of Michigan, Ann Arbor. Prior to this, he was with the Electrical and Computer Engineering Department, University of Wisconsin, Madison. He has also held industry positions with Xerox Corporation, Microchip Technology, and other companies, working in the area of integrated circuit design. His research interests include all aspects of design, fabrication, and packaging of micromachined sensors and actuators and their interface circuits. He has published approximately 130 papers in the field of MEMS, and has about 20 patents issued or pending.

Prof. Gianchandani is the recipient of a National Science Foundation Career Award. He serves on the editorial boards of Sensors and Actuators, IOP Journal of Micromechanics and Microengineering, and the Journal of Semiconductor Technology and Science. He also served on the steering and technical program committees for the IEEE/ASME International Conference on Micro Electro Mechanical Systems (MEMS) for many years, and served as a General Co-Chair for this meeting in 2002. At the University of Michigan, he serves as the Director of the College of Engineering Interdisciplinary Professional Degree Program in Integrated Microsystems.

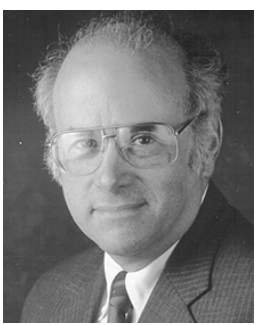

J. Leon Shohet (S'56-M'62-SM'72-F'78) received the Ph.D. degree from Carnegie Mellon University, Pittsburgh, PA, in electrical engineering in 1961.

He served on the faculty of The Johns Hopkins University, Baltimore, MD, before joining the faculty of the University of Wisconsin, Madison, in 1966 and was appointed Professor of Electrical and Computer Engineering in 1971. He is the Director of the Plasma Processing and Technology Laboratory and is the Founding Director of the University's NSF Engineering Research Center for Plasma-Aided Manufacturing, as well as the Past Chairman of the Department of Electrical and Computer Engineering. He is the author of two textbooks on plasma science, more than 150 journal articles, and more than 440 conference papers. He holds eight patents. His research interests include plasma-aided manufacturing; fusion, especially waves, instabilities, heating, confinement and diagnostics; communications; magnetohydrodynamics; electromagnetic field theory; biophysics; and nonlinear science.

Dr. Shohet is a Fellow of the American Physical Society. He received the Frederick Emmons Terman award of the American Society for Engineering Education, the Merit Award of the IEEE's Nuclear and Plasma Sciences Society, the IEEE Richard F. Shea Award, the IEEE Plasma Science Prize, the IEEE Centennial Medal and the John Yarwood Memorial Medal from the British Vacuum Council. Dr. Shohet founded the IEEE TRANSACTIONS ON PLASMA SCIENCE in 1973. 